\title{
Correlation analysis between the rate of respiration in the root and the active components in licorice (Glycyrrhiza uralensis)
}

\author{
PEIJUN GUO, ZHIRONG SUN, WENLAN LIU, LONG CHEN, YUAN DU and XINXIN WEI
}

School of Chinese Materia Medica, Beijing University of Chinese Medicine, Beijing 100029, P.R. China

Received July 9, 2013; Accepted October 30, 2013

DOI: 10.3892/etm.2013.1387

\begin{abstract}
The aim of this study was to investigate the correlation between root respiration and the percentage of active components in licorice (Glycyrrhiza uralensis Fisch.), in order to provide a foundation for the regulation and modulation of the quality of G. uralensis. Respiration efflux of annual and biennial $G$. uralensis was determined using a Li-7000 $\mathrm{CO}_{2} / \mathrm{H}_{2} \mathrm{O}$ analyzer. The root systems were scanned at a resolution of 3,000 dpi using an Epson Expression 10000XL scanner. Root growth was determined by analyzing the scanned images using WinRHIZO version Pro2007d software and the rate of respiration in the root was subsequently calculated. In addition, the percentages of the five major active components in licorice, glycyrrhizic acid, glycyrrhizin, isoliquiritin, liquiritigenin and isoliquiritigenin, were detected using high-performance liquid chromatography (HPLC). The correlation between the root respiration and the percentage of the active components was investigated. Significant seasonal changes were observed in the rates of respiration of first and zero-class roots. In annual and biennial $G$. uralensis, the maximum and minimum values for rate of respiration were present in July $(\mathrm{P}<0.05)$ and November $(\mathrm{P}<0.05)$, respectively. The correlation coefficients between the five major active components and the rate of respiration were -0.304 (glycyrrhizin), -0.129 (liquiritigenin), -0.441 (glycyrrhizic acid; $\mathrm{P}<0.05$ ), -0.471 (isoliquiritin; $\mathrm{P}<0.05$ ) and 0.148 (isoliquiritigenin). The percentages of glycyrrhizic acid and isoliquiritin were significantly negatively correlated with the rate of respiration in annual and biennial G. uralensis. Understanding the correlation between the root rate of respiration and the active components in G. uralensis may be beneficial to ensuring the quality of cultivated G. uralensis.
\end{abstract}

Correspondence to: Professor Zhirong Sun, School of Chinese Materia Medica, Beijing University of Chinese Medicine, No. 6 South Road, Beijing 100029, P.R. China

E-mail: zrs67@126.com

Key words: Glycyrrhiza uralensis, root respiration, active component, correlation analysis

\section{Introduction}

Licorice (Glycyrrhiza uralensis Fisch.), an ancient medicinal herb, has been frequently used in the medical, food processing and daily chemical industries. However, wild G. uralensis resources have been severely damaged and, in some places, are on the verge of extinction (1). For decades, the cultivation of G. uralensis in China has been proposed as a substitute for the wild resources. However, several problems, including poor production and unsatisfactory root quality, have been described. In particular, the percentage of glycyrrhizic acid in the cultivated G. uralensis has been measured to be $0.49-1.51 \%$ (1), which is less than the standard percentage $(>2 \%)$ set by the Pharmacopoeia of the People's Republic of China. Extensive studies have been performed to investigate the ability of water (2), nutrients (3) and seeding density (4) to increase the percentages of the active components in cultivated G. uralensis. However, no ideal conditions have been identified to enhance the percentages of the active components.

Root respiration has a significant impact on the primary productivity of plants (5). It has been demonstrated that $50-80 \%$ of the total net primary production is accounted for by the net primary production in the root (1). Högberg et al (6) revealed that $75 \%$ of the carbon allocated to the root was for respiration, which provided the energy for the growth, vital activity and the nutritional intake of the root (7). However, the effects of environmental and cultivation control factors on the synthesis and accumulation of the active components in G. uralensis remain unclear. In addition, no similar studies have been performed to evaluate the correlation between root respiration and the levels of the active components in G. uralensis. In this study, the aim was to analyze the fluctuations in root respiration and to investigate the correlation between root respiration and the five major active components (glycyrrhizic acid, glycyrrhizin, isoliquiritin, liquiritigenin and isoliquiritigenin) in G. uralensis. Glycyrrhizic acid has been widely used in the treatment of various liver diseases (8-14), while glycyrrhizin and isoliquiritin have been observed to exert antidepressive effects in mice (15). Liquiritigenin was demonstrated to prevent acute acetaminophen-induced liver injuries in rats (16) and isoliquiritigenin has been indicated to inhibit the growth and proliferation of various cancer cells and induce the apoptosis of cancer cells. In addition, isoliquiritigenin may promote the cellular proliferation of normal tissues (17).The results of this study may 
aid the cultivation of $G$. uralensis and promote the sustainable use of $G$. uralensis resources.

\section{Materials and methods}

Cultivation of $G$. uralensis. The G. uralensis seeds and seedlings were grown in Hangjingqi (Inner Mongolia, China). The plants were cultivated in April 2011 in a growth chamber in the Beijing University of Chinese Medicine (39 $55^{\prime} \mathrm{N}, 116^{\circ} 28^{\prime}$ $\mathrm{E}$, at an altitude of $54.7 \mathrm{~m})$. The plants were cultivated at an average temperature of $11.8^{\circ} \mathrm{C}$ and an annual rainfall of $577 \mathrm{~mm}$. The average evaporation capacity was $1,861 \mathrm{~mm}$, while the comparative humidity was $62 \%$.

The G. uralensis was grown in a polyvinyl chloride (PVC) tube, which was $5 \mathrm{~cm}$ above the ground. The tube was filled with sandy loam, containing organic matter $(0.286 \%)$, alkali-hydrolyzable nitrogen $(35.88 \mathrm{mg} / \mathrm{kg})$, soil-available phosphorus $(3.0 \mathrm{mg} / \mathrm{kg})$, soil-available potassium $(85.18 \mathrm{mg} / \mathrm{kg})$ and $\mathrm{CaCO}_{3}(2.71 \%)$. The $\mathrm{pH}$ value of the sandy loam was maintained at 7.87. The diameter and length of the tube were 30 and $80 \mathrm{~cm}$, respectively, and the bottom of the tube was sealed with gauze. The seeding and transplantation of the G. uralensis were conducted on May 10 each year. Four G. uralensis plants were cultivated in one PVC tube. The relative water content in the tube was maintained between 60 and 70\%. No fertilizer was given to the plants.

Determination of the rate of respiration. In order to evaluate the rate of respiration, the root was cleaned with distilled water and wrapped with wet gauze. The categorization of the root was performed as previously described (18). A zero-class root is also called an axial root and first-class roots mean the lateral roots growing on the axial root. Subsequently, the rate of respiration was calculated in accordance with the method from a previous study (19). To avoid wound respiration, Vaseline was smeared onto the section of the root system. The root was then placed in a $\mathrm{Li}-7000 \mathrm{CO}_{2} / \mathrm{H}_{2} \mathrm{O}$ analyzer ( $\mathrm{Li}-\mathrm{Cor}$, Lincoln, $\mathrm{NE}$, USA), in which the concentration of $\mathrm{CO}_{2}$ released by the root system was analyzed subsequent to constant airflow being obtained. In addition, the rate of respiration was calculated based on the $\mathrm{CO}_{2}$ flux.

Scanning of the root system. The root systems were scanned at a resolution of 3,000 dpi using an Epson Expression 10000XL scanner (Epson Co., Ltd., Long Beach, CA, USA). Root growth was measured by analyzing the scanned images using WinRHIZO version Pro2007d software (Regent Instruments Inc., Quebec Canada). All the experiments were performed at least six times.

Component evaluation of the root system. The samples were oven-dried at $50^{\circ} \mathrm{C}$ until a constant weight was achieved, prior to the roots being weighed and crushed. A 40-mesh screen was used as the threshold separating thick and fine roots. Subsequently, the active components of the G. uralensis were analyzed using a high-performance liquid chromatography (HPLC) assay with ultraviolet detection, based on a previous description (20). In brief, $5 \mathrm{ml} \mathrm{G}$. uralensis (0.01 g) was added to $5 \mathrm{ml}$ ethanol $(70 \%)$, prior to ultrasound extraction being performed at $40^{\circ} \mathrm{C}$ for $30 \mathrm{~min}(250 \mathrm{~W})$. Following cooling at

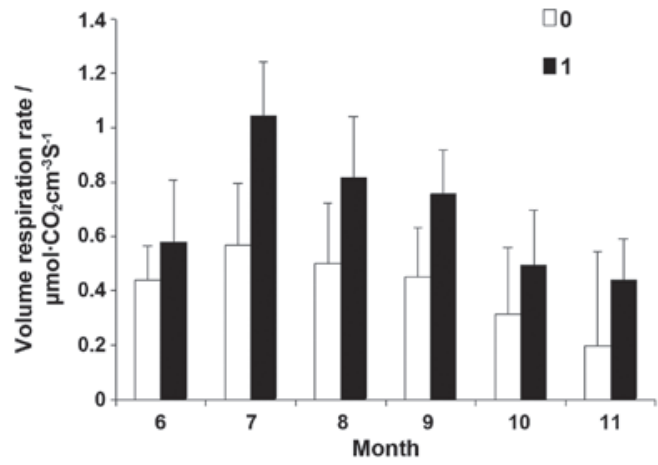

Figure 1. Changes in the root respiration of the annual licorice. White bars, zero-class roots; black bars, first-class roots.

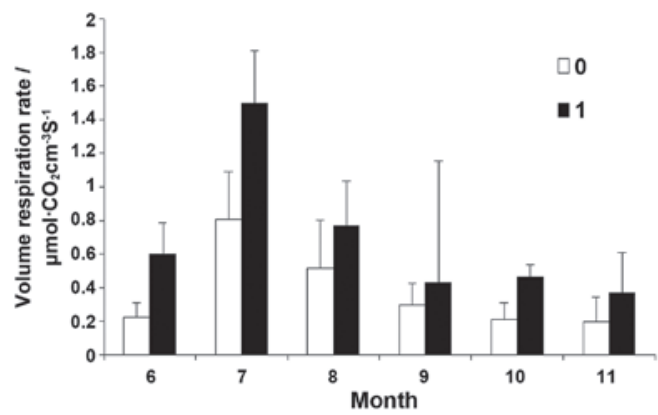

Figure 2. Changes in the root respiration of the biennial licorice. White bars, zero-class roots; black bars, first-class roots.

room temperature, $70 \%$ ethanol was added and the mixture was filtered using a Millipore filter with a diameter of $0.45 \mu \mathrm{m}$ (Millipore, Billerica, MA, USA). A chromatographic column (Diamonsil ${ }^{\circledR} \mathrm{C} 18,250 \times 4.6 \mathrm{~mm}, 5 \mu \mathrm{m}$; Agilent Technologies Inc., Santa Clara, CA, USA) was used for the HPLC analysis. A methyl cyanide-phosphoric acid mixture was used as the mobile phase. The wavelengths used for the analysis were as follows: $\lambda_{1}=237 \mathrm{~nm}, \lambda_{2}=365 \mathrm{~nm}$.

Statistical analysis. SPSS 19.0 statistical software (SPSS, Inc., Chicago, IL, USA) was used for the data analysis. $\mathrm{P}<0.05$ was considered to indicate a statistically significant difference.

\section{Results}

Changes in root respiration. In this study, samples of annual and biennial G. uralensis, harvested in June and November, 2011, were used to determine the rate of respiration. A significant difference was observed in the volume rate of respiration between the annual and biennial $G$. uralensis $(\mathrm{P}<0.05$, Figs. 1 and 2). In the annual and biennial $G$. uralensis, the maximum and minimum values for the rate of respiration were obtained in July $(\mathrm{P}<0.05)$ and November $(\mathrm{P}<0.05)$, respectively. Compared with the axial root, a marked increase in the rate of respiration was detected in the first-class root in the annual $(0.688$ versus $\left.0.375 \mu \mathrm{mol} \mathrm{CO} \mathrm{Cm}^{-3} \mathrm{sec}^{-1} ; \mathrm{P}<0.05\right)$ and biennial $(0.686$ versus $\left.0.411 \mu \mathrm{mol} \mathrm{CO}_{2} \mathrm{~cm}^{-3} \mathrm{sec}^{-1} ; \mathrm{P}<0.05\right)$ G. uralensis for months 6-11. These results were consistent with those obtained in a previous study by Ren et al (21). 
Table I. Percentages of the active components in G. uralensis root.

\begin{tabular}{lcccccc}
\hline G. uralensis & Root system & $\begin{array}{c}\text { Glycyrrhizin } \\
(\%)\end{array}$ & $\begin{array}{c}\text { Liquiritigenin } \\
(\%)\end{array}$ & $\begin{array}{c}\text { Glycyrrhizic acid } \\
(\%)\end{array}$ & $\begin{array}{c}\text { Isoliquiritin } \\
(\%)\end{array}$ & $\begin{array}{c}\text { Isoliquiritigenin } \\
(\%)\end{array}$ \\
\hline Annual & 0 & 0.50 & 0.07 & 1.61 & 0.09 & 0.02 \\
& 1 & 0.26 & 0.02 & 0.86 & 0.06 & 0.01 \\
\multirow{2}{*}{ Biennial } & 0 & 1.11 & 0.10 & 1.52 & 0.13 & 0.03 \\
& 1 & 0.54 & 0.04 & 0.98 & 0.08 & 0.02 \\
\hline
\end{tabular}

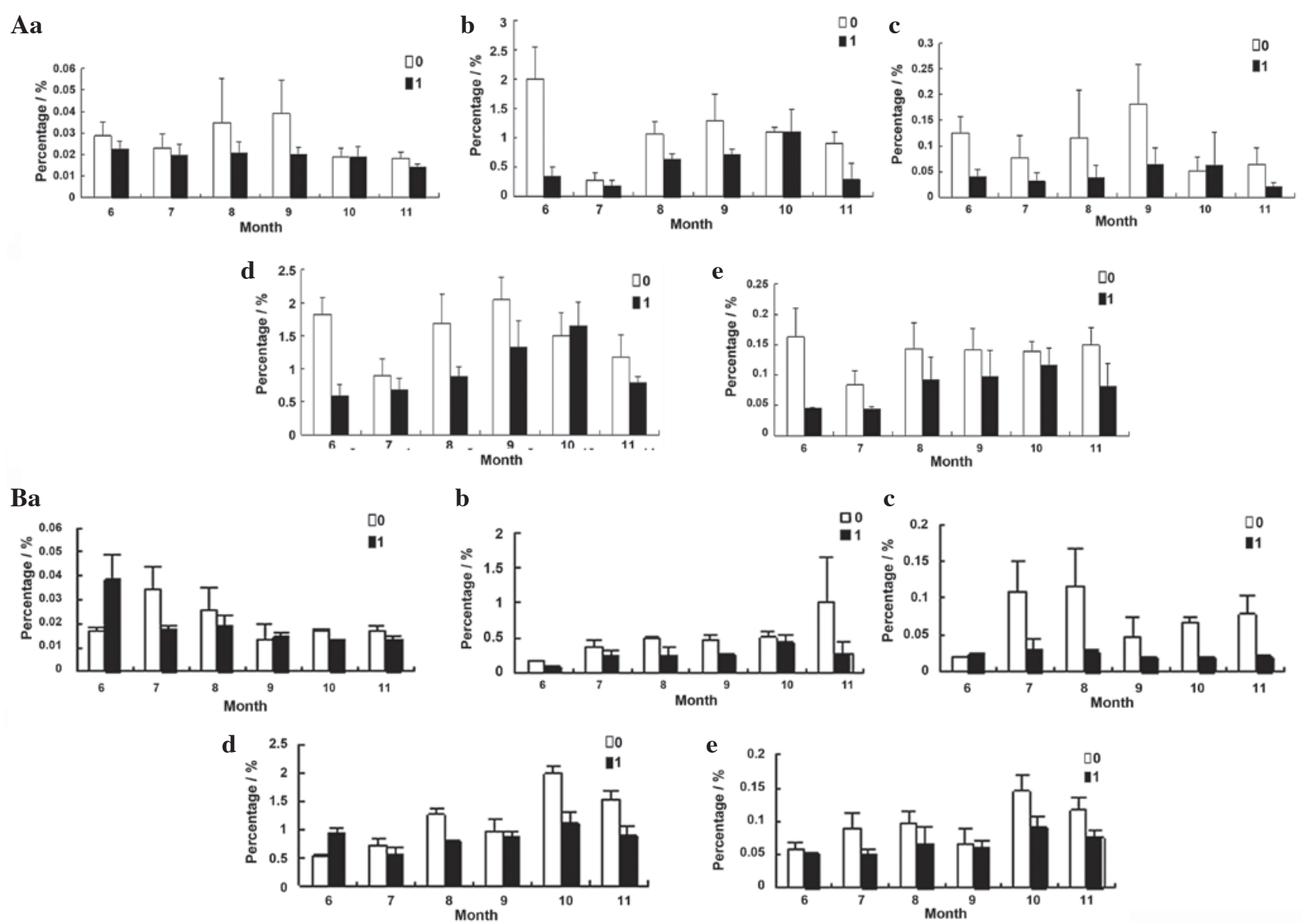

Figure 3. Seasonal comparisons of the five major active components in annual and biennial licorice. (A) Percentages of (Aa) glycyrrhizin, (Ab) liquiritigenin, (Ac) glycyrrhizic acid, (Ad) isoliquiritin and (Ae) isoliquiritigenin in annual licorice. (B) Percentages of (Ba) glycyrrhizin, (Bb) liquiritigenin, (Bc) glycyrrhizic acid, (Bd) isoliquiritin and (Be) isoliquiritigenin in biennial licorice. White bars, zero-class roots; black bars, first-class roots.

The maximum rate of respiration was affected by the soil temperature, water content and nutritional intake. In the present study, higher rates of respiration were observed in July and August, which was likely to be correlated with the soil temperature and root metabolism.

Fluctuation in active component levels. Five active components, specifically, glycyrrhizic acid, glycyrrhizin, isoliquiritin, liquiritigenin and isoliquiritigenin, were analyzed in G. uralensis in our study.

Fig. 3 shows the fluctuations in the percentages of glycyrrhizin, liquiritigenin, glycyrrhizic acid, isoliquiritin and isoliquiritigenin present in the G. uralensis harvested in June and
November, respectively. The data shown in the figure indicate that significant fluctuations occurred in the percentages of the five major components $(\mathrm{P}<0.05)$, depending on the season. The percentage of glycyrrhizic acid measured in the axial root in the present study was consistent with the value obtained in a previous study (3). Furthermore, the maximum percentage of glycyrrhizic acid in annual G. uralensis was apparent in September, which was also consistent with a previous investigation (22).

As shown in Table I, the levels of the five major components in the axial roots were higher than those of the first-class roots. In addition, the percentages of glycyrrhizin, isoliquiritin, liquiritigenin and isoliquiritigenin were higher in the biennial $G$. uralensis than in the annual G. uralensis. 
Table II. Correlation between root respiration and the content of the active components in G. uralensis.

\begin{tabular}{|c|c|c|c|c|}
\hline Component & Growth year & Fitting equation & $\mathrm{R}^{2}$ & $\mathrm{r}$ \\
\hline \multirow[t]{3}{*}{ Glycyrrhizin } & Annual G. uralensis & $Y=-0.2461 x+0.5166$ & 0.1409 & -0.375 \\
\hline & Biennial G. uralensis & $Y=-1.1270 x+1.4478$ & 0.2350 & -0.485 \\
\hline & Mixture & $Y=-0.4695 x+0.8611$ & 0.0921 & -0.304 \\
\hline \multirow[t]{3}{*}{ Liquiritigenin } & Annual G. uralensis & $Y=-0.0031 x+0.0500$ & 0.0010 & -0.032 \\
\hline & Biennial G. uralensis & $Y=-0.0620 x+0.1070$ & 0.0932 & -0.305 \\
\hline & Mixture & $Y=-0.0183 x+0.0706$ & 0.0167 & -0.129 \\
\hline \multirow[t]{3}{*}{ Glycyrrhizic acid } & Annual G. uralensis & $Y=-0.5873 x+1.3244$ & 0.2680 & -0.518 \\
\hline & Biennial G. uralensis & $Y=-0.9622 x+1.7805$ & 0.2016 & -0.449 \\
\hline & Mixture & $Y=-0.6793 x+1.4993$ & 0.1942 & $-0.441^{\mathrm{a}}$ \\
\hline \multirow[t]{3}{*}{ Isoliquiritin } & Annual G. uralensis & $Y=-0.0353 x+0.0995$ & 0.2030 & -0.451 \\
\hline & Biennial G. uralensis & $Y=-0.1226 x+0.1754$ & 0.4811 & $-0.694^{\mathrm{a}}$ \\
\hline & Mixture & $Y=-0.0582 x+0.1259$ & 0.2215 & $-0.471^{\mathrm{a}}$ \\
\hline \multirow[t]{3}{*}{ Isoliquiritigenin } & Annual G. uralensis & $Y=0.0063 x+0.0169$ & 0.0739 & 0.272 \\
\hline & Biennial G. uralensis & $Y=-0.0028 x+0.0247$ & 0.0739 & -0.086 \\
\hline & Mixture & $Y=0.0039 x+0.0196$ & 0.0218 & 0.148 \\
\hline
\end{tabular}

${ }^{\mathrm{a}} \mathrm{P}<0.05$.

Correlation between root respiration and the levels of the active components in G. uralensis. Table II summarizes the correlation between root respiration and the percentages of the active components in G. uralensis. A previous study indicated that the rate of respiration in the root increased as the nitrogen concentration in the root increased (23). In general, the first-class root exhibited the maximum content of nitrogen and rate of respiration. In plants, $\sim 90 \%$ of nitrogen has been shown to be present in the form of various proteins (24). The levels of glycyrrhizic acid and glycyrrhizin have been demonstrated to be negatively correlated with primary metabolites, including soluble protein, in the roots (25). Therefore, compared with the axial root, a higher nitrogen content and rate of respiration and a lower secondary metabolite content were apparent in the first-class root.

\section{Discussion}

The rates of respiration in the axial and first-class roots of annual and biennial G. uralensis showed significant fluctuations in the different seasons. The rates of respiration were notably higher in July and were predominantly lower in November. In the present study, it was identified that the rate of respiration was negatively correlated with the levels of glycyrrhizin, liquiritigenin, glycyrrhizic acid and isoliquiritin, while isoliquiritigenin was positively correlated with the rate of respiration. Based on these results, it may be possible to enhance the accumulation of active components by modulating the rate of respiration in G. uralensis. To the best of our knowledge, the synthesis and accumulation of the secondary metabolites was affected by air (26), sunlight (1), water (27), soil nutrition (28) and temperature (29). In addition, the root respiration was affected by atmospheric $\mathrm{CO}_{2}$ concentration (23), soil temperature (27), soil nutrition $(24,25)$ and water content in the soil (30). In the present study, the root respiration was negatively correlated with the levels of the major active components in $G$. uralensis, demonstrating that it may be possible to alter the percentage of the active components by modulating the root respiration.

It has been demonstrated that the rate of respiration in the root is positively correlated with the root tissue nitrogen concentration (31). Therefore, the nitrogen concentration in the root may be used to evaluate the root respiration, providing greater accuracy than biomass (32). In the present study, the levels of glycyrrhizic acid and isoliquiritin were significantly correlated with the rate of respiration, with correlation coefficients of -0.441 and -0.471 , respectively. This indicated that glycyrrhizic acid and isoliquiritin may be used as indices for the evaluation of root respiration.

Wild G. uralensis resources have been severely damaged in a number of places due to over-excavation. Although biotechniques have been proposed for the cultivation of the G. uralensis, the results have been controversial (33). To meet the demands for $G$. uralensis, it is crucial to identify the optimal cultivation conditions. Wang et al (15) suggested that agronomic measures were important for the cultivation of G. uralensis (15). In addition, it has been indicated that increased nutrition and water content may attenuate the percentage of glycyrrhizic acid (34). However, the correlation between the levels of active components and environmental factors remain poorly defined.

In order to expand the G. uralensis resources, biotechniques have been used to produce the plant. However, the results have not been consistent (35) and the techniques have not been used in practice. Our study showed that the root respiration was significantly negatively correlated with the levels of glycyrrhizic acid and isoliquiritin. In addition, the root respiration may affect the synthetic pathway of active components in G. uralensis. 


\section{Acknowledgements}

This study was supported by a grant from the Beijing University of Chinese Medicine (no. 2009JYB22-JSO35).

\section{References}

1. Edwards NT, Shugart HH Jr, McLaughlin SB, Harris WF and Reichle DE: Carbon metabolism in terrestrial ecosystems. In: Dynamic Properties Of Forest Ecosystems. DE Reichle (ed). Cambridge University Press, Cambridge, pp499-536, 1980.

2. Cao JM: Research on adaptation mechanism of Glycyrrhiza uralensis to water deficiency. Journal of Anhui Agricultural Sciences 37: 7504-7505, 2009 (In Chinese).

3. Zhang Y, Wang JY, Liu Y and Wang WQ: Nitrogen nutrition in Glycyrrhiza uralensis Fisch. Journal of Beijing Forestry University 27(3): 57-60, 2005 (In Chinese).

4. Sun ZR, Zhai MP, Wang WQ and Li YR: Effects of density on seedling growth and glycyrrhizinic acid content in Glycyrrhiza uralensis. Zhongguo Zhong Yao Za Zhi 32: 2222-2226, 2007 (In Chinese)

5. Atkin OK, Edwards EJ and Loveys BR: Response of root respiration to changes in temperature and its relevance to global warming. New Phytol 147: 141-154, 2000.

6. Högberg P, Nordgren A and Ågren GI: Carbon allocation between tree root growth and root respiration in boreal pine forest. Oecologia 132: 579-581, 2002.

7. Hou JL, Li WD, Zheng QY, Wang WQ, Xiao B and Xing D: Effect of low light intensity on growth and accumulation of secondary metabolites in roots of Glycyrrhiza uralensis Fisch. Biochem Syst Ecol 38: 160-168, 2010.

8. Guo J, Wang JY and Koo MWL: Anti-oxidative effect of glycyrrhizin on acute and chronic $\mathrm{CCl}_{4}$-induced liver injuries. J Gastroen Hepatol 21 (Suppl 2): A154, 2006.

9. Zhai D, Zhao Y, Chen X, Guo J, He H, Yu Q, Yang J, Davey AK and Wang J: Protective effect of glycyrrhizin, glycyrrhetic acid and matrine on acute cholestasis induced by alpha-naphthyl isothiocyanate in rats. Planta Med 73: 128-133, 2007.

10. Tang B, Qiao H, Meng F and Sun X: Glycyrrhizin attenuates endotoxin-induced acute liver injury after partial hepatectomy in rats. Braz J Med Biol Res 40: 1637-1646, 2007.

11. Abe K, Ikeda T, Wake K, Sato T and Inoue H: Glycyrrhizin prevents of lipopolysaccharide/D-galactosamine-induced liver injury through down-regulation of matrix metalloproteinase- 9 in mice. J Pharm Pharmacol 60: 91-97, 2008.

12. Armanini D, Mattarello MJ, Fiore C, Bonanni G, Scaroni C, Sartorato $\mathrm{P}$ and Palermo M: Licorice reduces serum testosterone in healthy women. Steroids 69: 763-766, 2004

13. Lee CH, Park SW, Kim YS, Kang SS, Kim JA, Lee SH and Lee SM: Protective mechanism of glycyrrhizin on acute liver injury induced by carbon tetrachloride in mice. Biol Pharm Bull 30: 1898-1904, 2007.

14. Yoshida T, Abe K, Ikeda T, Matsushita T, Wake K, Sato T and Inoue H: Inhibitory effect of glycyrrhizin on lipopolysaccharide and d-galactosamine-induced mouse liver injury. Eur J Pharmacol 576: 136-142, 2007.

15. Wang W, Hu X, Zhao Z, Liu P, Hu Y, Zhou J, Zhou D, Wang Z, Guo D and Guo H: Antidepressant-like effects of liquiritin and isoliquiritin from Glycyrrhiza uralensis in the forced swimming test and tail suspension test in mice. Prog Neuropsychopharmacol Biol Psychiatry 32: 1179-1184, 2008.

16. Kim YW, Ki SH, Lee JR, Lee SJ, Kim CW, Kim SC and Kim SG: Liquiritigenin, an aglycone of liquiritin in Glycyrrhizae radix, prevents acute liver injuries in rats induced by acetaminophen with or without buthionine sulfoximine. Chem Biol Interact 161:125-138, 2006.
17. Gao X, Wang W, Wei S and Li W: Review of pharmacological effects of Glycyrrhiza radix and its bioactive compounds. Zhongguo Zhong Yao Za Zhi 34: 2695-2700, 2009 (In Chinese).

18. Fitter AH: Morphometric analysis of root systems: application of the technique and influence of soil fertility on root development in two herbaceous species. Plant Cell Environ 5: 313-322, 1982.

19. Lee MS, Nakane K, Nakatsubo T and Koizumi H: Seasonal changes in the contribution of root respiration to total soil respiration in a cool-temperate deciduous forest. Plant Soil 255: 311-318, 2003.

20. Zhou XZ: The study of rapid quality analysis method of licorice by UV-vis and infrared spectrophotometry. Beijing: Beijing University of Chinese Medicine: pp 30-36, 2012 (In Chinese).

21. Ren J, Xu CY, Wei YB,Lin YM and Duan YH: Seasonal dynamics and influence factor of root respiration of Fraxinus mandushurica in the Changbai Moutain. Lin Ye Ke Xue 46: 77-83, 2010 (In Chinese).

22. Zhao ZH, Cao JG, Fu YJ, Tang ZH and Zu YG: Distribution characteristics of glycyrrhizic acid in wild and cultivated liquorices and their applications. Zhiwuxue Tongbao 23: 164-168, 2006 (In Chinese).

23. Li CD, Dong HR and Li JC: Influence of various ratios of nitrogen nutrition on photosynthetic and sugar metabolism of cotton. Mian Hua Xue Bao 15: 87-90, 2003 (In Chinese).

24. Olsthoorn AFM, Keltjens WG, van Baren B and Hopman MCG: Influence of ammonium on fine root development and rhizosphere $\mathrm{pH}$ of Douglas-fir seedlings in sand. Plant Soil 133: 75-81, 1991.

25. Wang DF: Regulation of agronomic measures on the growth and development of Glycyrrhiza uralensis. Lanzhou: Gansu Agricultural University. pp45-55, 2008.

26. Maier CA and Kress LW: Soil $\mathrm{CO}_{2}$ evolution and root respiration in 11 year-old loblolly pine (Pinus taeda) plantations as affected by moisture and nutrient availability. Can J For Res 30: 347-359, 2000.

27. Zogg GP, Zak DR, Burton AJ and Pregitzer KS: Fine root respiration in northern hardwood forest in relation to temperature and nitrogen availability. Tree Physiol 16: 719-725, 1996.

28. Zhang J, Shen YB and Xu CY: Reviews on root respiration and it responses to environment changes. Journal of Northeast Forestry University 35(2): 78-81, 2007 (In Chinese).

29. Burton AJ and Pregitzer KS: Field measurements of root respiration indicate little to no seasonal temperature acclimation for sugar maple and red pine. Tree Physiol 23: 273-280, 2003.

30. Chen DH, Ye HC, Li GF and Liu Y: Advances in molecular biology of plant isoprenoid metabolic pathway. Act Bot Sin 42: 551-558, 2000.

31. Desrochers A, Landhäusser SM and Lieffers VJ: Coarse and fine root respiration in aspen (Populus tremuloides). Tree Physiol 22: 725-732, 2002.

32. Ryan MG, Hubbard RM, Pongracic S, Raison RJ and McMurtrie RE: Foliage, fine-root, woody-tissue and stand respiration in Pinus radiata in relation to nitrogen status. Tree Physiol 16: 333-343, 1996.

33. Henry M, Edy AM and Marty B: Isolation of licorice protoplasts (Glycyrrhiza glabra L. var. typica Reg. et Hed) from cell suspension cultures not producing glycyrrhetinic acid. C R Acad Sci III 299: 899-903, 1984 (In French).

34. Zhao ZH, Yu JH, Yang FJ, Zu YG and Cao JG: Influences of artificial disturbance degrees on the contents of Glycyrrhizic acid and flavonoids in different parts of Glycyrrhiza uralensis. Sheng Tai Xue Bao 24: 2799-2803, 2004 (In Chinese).

35. Cao HL and Zhou YQ: Review of the influencing factor of glycyrrhizic acid content in Glycyrrhiza uralensis Fisch. Modern Chinese Medicine 11(2): 6-10, 2009 (In Chinese). 